\section{Botulinum neurotoxin type $A$ in neurology: update}

\author{
Marco Orsini,1,2 Marco Antonio Araujo \\ Leite,2 Tae Mo Chung, 3 Wladimir Bocca,4 \\ Jano Alves de Souza, ${ }^{3}$ Olivia Gameiro de \\ Souza, ${ }^{3}$ Rayele Priscila Moreira,5 Victor \\ Hugo Bastos, 5 Silmar Teixeira, 5 Acary \\ Bulle Oliveira, 4 Bruno da Silva Moraes, 1 \\ André Palma Matta,2 Luis Jorge Jacinto5 \\ 1 Centro Universitário Augusto Motta, \\ Bonsucesso, Rio de Janeiro; \\ 2Universidade Federal Fluminense, \\ Departamento de Neurologia, Niterói, \\ Rio de Janeiro; 3Universidade de São \\ Paulo - USP, São Paulo; 4Universidade \\ Federal de São Paulo - UNIFESP, São \\ Paulo, 5Universidade Federal do Piaui, \\ Parnaíba, Brasil
}

\section{Abstract}

This paper reviews the current and most neurological (central nervous system, CNS) uses of the botulinum neurotoxin type A. The effect of these toxins at neuromuscular junction lends themselves to neurological diseases of muscle overactivity, particularly abnormalities of muscle control. There are seven serotypes of the toxin, each with a specific activity at the molecular level. Currently, serotypes A (in two preparations) and B are available for clinical purpose, and they have proved to be safe and effective for the treatment of dystonia, spasticity, headache, and other CNS disorders in which muscle hyperactivity gives rise to symptoms. Although initially thought to inhibit acetylcholine release only at the neuromuscular junction, botulinum toxins are now recognized to inhibit acetylcholine release at autonomic cholinergic nerve terminals, as well as peripheral release of neurotransmitters involved in pain regulation. Its effects are transient and nondestructive, and largely limited to the area in which it is administered. These effects are also graded according to the dose, allowing individualized treatment of patients and disorders. It may also prove to be useful in the control of autonomic dysfunction and sialorrhea. In over 20 years of use in humans, botulinum toxin has accumulated a considerable safety record, and in many cases represents relief for thousands of patients unaided by other therapy.

\section{Introduction}

Botulinum toxin type A treatment has already made a dramatic impact on neurologic clinical practice to provide a new effective treatment and management of some diseases. It's a bacterial endotoxin produced by Clostridium botulinum during growth and reproduction. According to the difference in toxin antigenic profiles, it is classified into seven serotypes namely type A, B, C, D, E, F and G. Type A, B, E and F are human toxic types while type $C$ and $D$ are animal and poultry toxic types. ${ }^{1}$ Botulinum toxin acts as the neuromuscular junction to inhibit the release of neurotransmitter, acetylcholine, presynaptically by the following steps: i) fast, specific and irreversible binding to acceptors on presynaptic nerve surface; ii) uptake of toxin into cell vesicle; iii) translocation of toxin into cytosol across vesicle membrane, iv) toxin activates proteolysis which, in turn, blocks the release of acetylcholine. In recent years, other possible mechanisms of botulinum toxin have also been studied including its actions on afferent nerve, nociceptive sensory nerve and parasympathetic neuron. These studies serve as basis evidence that help develop innovative indications and new therapeutic applications. Botulinum toxin (BT) is currently used in those entities characterized by excessive muscle contraction, including dystonia and spasticity. In addition, BT has been used to control pain associated with increased muscle contraction in dystonia and spasticity, but also is useful to control chronic pain not associated with muscle contraction, such as chronic daily headache. Finally, BT is useful in sialorrhea.2,3

Neurological disorders that trigger spasticity as Stroke, Multiple Sclerosis, Head Trauma, Spinal Cord Trauma, Cerebral Palsy and some motor neuron diseases (Spastic Paraparesis, Amyotrophic Lateral Sclerosis), can be managed by applying botulinum toxin. The mechanism of action is complex, mainly acting on terminal neuromuscular junction, but also exhibiting analgesic properties, probably through inhibition of pain neurotransmitters release. ${ }^{1}$ Note: In addition to the direct action on striated muscle, botulinum toxin may act in the body of the muscle spindle and reduce centripetal conduction of information. ${ }^{2}$

\section{Injections of botulinum toxin in spasticity}

\section{Understanding the pathophysiolo- gy of spasticity}

Spasticity is triggered on the occurrence of
Correspondence: Marco Antonio Araujo Leite, Departamento de Neurologia, Universidade Federal Fluminense, Tavares de Macedo, 95, 902, Icarai, Niterói, RJ, Brasil. E-mail: maaraujoleite@yahoo.com.br

Key words: botulinum toxin; neurology; rehabilitation.

Contributions: the authors contributed equally.

Conflict of interest: the authors declare no potential conflict of interest.

Received for publication: 25 February 2015. Accepted for publication: 17 August 2015.

This work is licensed under a Creative Commons Attribution NonCommercial 3.0 License (CC BYNC 3.0).

(C) Copyright M. Orsini et al., 2015

Licensee PAGEPress, Italy

Neurology International 2015; 7:5886

doi:10.4081/ni.2015.5886

an imbalance between facilitatory and inhibitory input of alpha motor neurons leading to motor system hyperexcitability. In other words, is a motor disorder marked by the increase of tonic stretch reflex-speed dependent. In association with increased tonus, other signs of involvement of the pyramidal system as tendon hyperreflexia, clonus and presence of Babinski's sign may occur. Patients affected by spasticity commonly mention complaints such as muscle stiffness, impaired dexterity in performing functional tasks, joint instability, painful muscle contractures, spasms, limited range of motion and abnormal postures. ${ }^{4}$

\section{Assessment of spasticity in clinical practice}

In the assessment of spasticity, profiles, measurements and indicators of evaluation should be individualized and based on the International Classification of Functioning, Disability and Health (ICF) of the World Health Organization (WHO). 5 The Modified Ashworth Scale is undoubtedly the most widely used instrument for the assessment of spasticity due to its reliability and reproducibility. With it, the passive movement of the segment evaluated is performed aiming to quantify the degree of resistance to movement. The scale is ordinal, with values ranging from 0 to $4 .{ }^{6}$ The Barthel index and the Functional Independence Measure (FIM) are used to measure the level of functional independence of patients in their basic and instrumental activities of daily living. 7,8

Currently the gait labs are able to assess in greater detail the performance of patients (post-application of botulinum toxin) by distance traveled, speed and amplitude of steps. 
The analysis must be carried through standardization of physical examination and gait pattern records in two-dimensional video recording. They should, if available in the services, be applied the three-dimensional kinematic and kinetic records, the dynamic electromyography, baropodography and measurement of energy consumption during gait. ${ }^{9}$

\section{Types off botulinum toxin type $A$, points of application, ways of dilu- tion and long term effects}

Currently, the therapeutic preparations of the most common TB type A for the treatment of patients with neurological injuries are Botox® (Alergan Inc., Irvine, CA, EUA) and/or Dysport ${ }^{\circledR}$ (Ipsen Ltd., Slough, Berks, RU, USA). There are other types of therapeutic preparations not commented in this chapter.

We alert that doses are not interchangeable between the different preparations of botulinum toxin type A. Furthermore, they vary according to the selected muscles. The main dose modifiers are muscle mass, degree of spasticity (Ashworth Scale) and results in earlier applications. Unpreserved physiological solution (sodium chloride $0.9 \%$ ) is used for dilution. The number of points of application is dependent on the morphology of muscle groups and is usually based on: i) the surface anatomy; ii) electromyography; iii) electrical stimulation; iv) ultrasound (deeper muscle). ${ }^{10,11}$

The onset of botulinum toxin efficacy ranged from few days to few weeks after injection. Toxin induces muscle paralysis and clinically atrophy. Efficacy duration lasts for 2-4 months or even longer. Muscle shows response to botulinum toxin treatment for a long time except when formation of neutralizing antibody occurs. It is noteworthy that the efficacy of botulinum toxin could be maximized when combining with physiotherapy and orthopedic devices, such as, orthoses. Some factors may limit the benefits of botulinum toxin, which are: the absence of a rehabilitation program or inappropriate treatment after the application; the presence of fixed contractures; patients with cognitive impairments and antibodies against botulinum toxin are some of them. ${ }^{12}$

Spasticity may also prevent severe muscle atrophy manage loss of bone mass and edema, as well as the risk of deep venous thrombosis. As a result, these factors must be analyzed thoroughly before application. Conditions such as bedsores, urinary tract infection, deep vein thrombosis, fecal impaction, emotional stress, fractures, and dislocations among others may cause a momentary increase of spasticity. After clinical resolution of these problems, the patients present spontaneous attenuation of the tonus without any intervention. ${ }^{13}$

\section{Botulinum toxin use in spasticity}

\section{Stroke}

In these patients the pattern of spasticity is predominant in the flexor muscles of the upper limbs, with posture in adduction and internal rotation of the shoulder, elbow flexion, pronation of the wrist and finger flexion. In the lower limbs, spasticity predominates in the extensor muscles, with extension and internal rotation of the hip, knee extension, plantar flexion and foot inversion. This characteristic posture receives the name of Wernicke-Mann attitude. 14,15 We alert that in some cases, such pattern is not present. Therefore, before any procedure, a careful/judicious review should be performed. Such doses may vary according to the bibliography used.

\section{Spastic paraparesis}

Although patients with neuromuscular diseases such as, for example, in spastic paraparesis due to multiple causes, are considered as relative contraindication to the use of botulinum toxin, some may benefit from therapy. The major problem in these patients is the presence of chronic and progressive weakness of the muscles of the lower limbs, often causing the pattern of extensor spasticity to be useful to the performing of certain functional activities. When treatment of spasticity is really necessary, the therapeutic target is the adductor muscles of the lower limbs. The application of botulinum toxin may facilitate the gait patterns and assist patients in hygiene, changes in position, transfers and other tasks. The dose varies according to the degree of spasticity and therapeutic target. 16

\section{Amyotrophic lateral sclerosis}

In amyotrophic lateral sclerosis (ALS), countless studies demonstrate the effectiveness of botulinum toxin injection for the management of sialorrhea, dysphagia and pain. ${ }^{17,18}$ The therapy can also be used with caution in those cases where spasticity is accentuated, impairing caregivers in performing activities related to hygiene and changes in position $(\mathrm{XXX})$. It is important to emphasize that there are cases of patients with ALS who developed generalized weakness after focal botulinum toxin injection. ${ }^{19}$ Professionals should be careful in the evaluation and in the possible damages after application. As previously mentioned, the presence of spasticity can be a useful strategy for implementing certain functions in patients with ALS.

\section{Chronic childhood encephalopathy}

Cerebral palsy as an acquired or geneticrelated condition is the most common isolated cause of spasticity and the most studied disor- der linked to botulin neurotoxin therapy use, mainly in hemiplegia and spastic diplegia forms. Clinical use of BoNT-A provides many different benefits, including a better control in pain, muscle spasms, functional recovery and prevention of severe contractures. ${ }^{20}$ Children with chronic non progressive childhood encephalopathy were evaluated in many different studies about performance of BoNT-A injections for spasticity in the lower and upper limbs. ${ }^{21,22}$ A recent meta-analysis was performed and disclosed a short-term effective result for lower limb muscle tone, in passive ankle range of motion and gait speed, and a late-onset effectiveness on gross motor function measures for lower limbs. ${ }^{22}$ These cases were studied after BoNT-A injections in ankle flexors (including soleus and gastrocnemius), knee flexors and hip adductors. Despite the fact that lower limb involvement is more common in cerebral palsy, in some cases spastic quadriparesisis an important clinical finding and should care attention.

A recent randomized double-blind clinical trial was also performed to evaluate the safety and efficacy of BoNT-A in different degrees of upper extremity spasticity and showed shortterm improvements in function with a well-tolerated and safe profile, irrespective of the individual spasticity pattern or degree. ${ }^{21}$ There are many protocols of multiple injection sessions described in the literature without a clear benefit from one to the others. There is also no evidence that low or high doses improve spasticity in significantly different degrees.

\section{Other neurological diseases and/or clinical manifestations}

\section{Injections of botulinum toxin type A in cervical dystonia}

Most patients with cervical dystonia present with cosmetic, social and pain complaints frequently originate a common search for medical care. Although it can occur in association with complex movement disorder phenotypes (including hemifacial spasms, blepharospasms, Meige syndrome, or mandibular dystonia and spasmodic dysphonia), most cases present isolated cervical dystonia picture, even the fracture cases. Clinical trials have been conducted since the 1980 and almost always provided important evidence regarding disease symptom improvement after botulinum toxin use as a long-term efficient and safety therapeutic method. However, there is little data regarding the role of the long-term effects of the injections on neuroplasticity and peripheral and central neuromodulatory mechanism sexist.23-25 


\section{Injections of botulinum toxin type A} in tension headache and migraine

Many studies regarding the clinical therapeutic use of botulinum toxin for prophylactic treatment of migraine, chronic daily headache, chronic or persistent tension-type headache and chronic cervicogenic headache have been performed. However, a few provide conclusive and definitive data of its efficacy and reliable use. Most important clinical data have been originated in studies regarding its use for chronic migraine and, at some extention it can be applied to the different groups of headaches. ${ }^{26}$ In cases of refractory chronic migraine and in patients which discontinued oral prophylactics, there is increasing evidence that toxin injection can provide important therapeutic improvement. The most relevant studies showed that botulinum toxin $\mathrm{A}$ use was associated with moderate benefit in chronic daily headaches and chronic migraines, without an evidence of fewer episodic migraine occurrence or a decrease in chronic tension-type headache episodes per month. Despite the well-tolerated and low adverse effect profile in cases of headache, few medical centers have experienced teams in technical application and provide adequate patient outcomes.23,27

\section{Injections of botulinum toxin type A in sialorrhoea}

Inherited and acquired neuromuscular and neurodegenerative causes of dysphagia are frequently related to severe compromise of quality. Pulmonary infections and sialorrhea represents one of the most devastating symptoms linked to swallowing disorders. ${ }^{23,28}$ The benefits from clinical use of botulinum toxin have been widely proved to improve drooling severity in neuromuscular patients with moderate and severe dysphagia and sialorrhea, ${ }^{29}$ especially in cases of motor neuron disease (like Amyotrophic lateral sclerosis) and when applied in association with different therapeutic options, such as topical and oral anticholinergic drugs and phonotherapy. It is also clear in cases of dysphagia that the higher the dose injected in the parotid and submandibular glands, the stronger the effects and the more common the side effects. There is no evidence of the most appropriate techniques and esthetic drugs.

\section{Absolute and relative contraindica- tions to the use of botulinum toxin type $\mathrm{A}$}

Although its relative well-tolerated and safety therapeutic use, physicians must be aware of the most important absolute and relative contraindications of botulinum toxin type A use. Absolute contraindications include the existence of some relevant neuromuscular disorders (like neuromuscular junction diseases, including autoimmune acquired myasthenia gravis and Eaton-Lambert syndrome), social and psychological instability (as there is no proper social support in cases of lack of success), pregnancy, lactating females, skin or muscle local infection, and the history of adverse effects linked to previous toxin use. The most common relative contra indication is a fixed contracture of an involved joint to be injected. Recent botulinum neurotox application in less than 3 months is also a relative contra indication as the common finding of neutralizing antibodies comes more evident in such condition. Important attention must be carried in cases with severe late-stage motor neuron disease or polyneuropathies. ${ }^{23,30}$ As previously disclosed in this text, therapeutic use of drugs affecting the neuromuscular transmission should also be avoided, unless extremely necessary representing thus, a relative contra indication.

\section{Physical medicine and rehabilita- tion after application of botulinum} toxin type $\mathrm{A}$

Different definitions of spasticity have been published, nevertheless it's very important to keep in mind that, what patients really present is not pure spasticity but a number of types of muscle hyperactivity that co-exist and sometimes are not easy to differentiate. According to the most recent and worldwide recognized guidelines, patients with spasticity should be treated by multidisciplinary teams that are knowledgeable and experienced in the field, and the treatment should be individualized and is to include different modalities or techniques of physical medicine and rehabilitation.

This is not a universally accepted definition of Spasticity and different authors have proposed others: Muscle hyperactivity, Reversible muscle hypertonia, $, 31,32$ to name a couple of them. It's presentation can be variable, depending on the extent, topography and time since occurrence of the lesion(s). Whatever the form of presentation is, it's due to involuntary muscle hyperactivity and frequently leads to pain, abnormal postures and impaired function. Even when it's due to static lesions, location and severity of spasticity may change over time. Therefore, the patient needs to be assessed repeatedly and management should be adjusted accordingly.

\section{Spasticity management}

To try and describe thoroughly the effects of spasticity on a person's life, we should resort to the World Health Organization International Classification of Functioning, Disability and Health (ICF), which describes the effects of any health condition on: body functions and structures, impairment; ability to perform tasks, activities; involvement in society (citizenship), participation. For spasticity management, the treatment goals must aim to promote improvements on impairments but also in task performance: mobility, carrying, moving and handling objects, or, self care and domestic life activities, decrease in care-burden, facilitate handling/physical therapies and positioning, maintaining joint range of motion, avoiding skin complications, improving cosmetics and self-esteem. It's been well documented that spastic muscles suffer structural changes, the so-called non neurogenic components of spasticity. That's why, if untreated, it can lead to fixed contractures and permanent restriction of joint movement, resulting in rapidly progressive limb deformity.

\section{Differential diagnosis}

Severe spasticity is often difficult to differentiate from fixed contractures. General anesthesia, intravenous sedation or local nerve blocks can be used to differentiate these two conditions. These interventions temporarily switch off'muscle hyperactivity and hence, allow an evaluation of the degree of contracture present.

\section{Assessement of spasticity}

To assess spastic patients we must resort to a number of different tools, according to our expertise, human and technical resources and experience. Fundamental are: i) clinical scales: modified Ashworth scale (muscle tone), Tardieu scale (velocity dependent component), clonus, spasm frequency scale, associate reaction rating scale, pendulum test, tonic inhibition; ii) neurophysiological methods, EMG, H-reflex; iii) biomechanical methods: goniometry,muscle resistance. Of course we must evaluate more than just the spasticity aspect of a neurologic patient, before we can design a treatment strategy. The global assement should include also: i) goniometry, muscle strength, selective residual motor control; ii) quantifying symptoms: visual/numerical Analogic Scales, Lickert Scales, Shoulder Q, Spin; iii) Functional Scales: 1) Upper Limbs: 9hole peg test; Frenchay arm test; PRS; FuglMeyer; ARMA; Action Research Arm Test; Rivermead Motor Assessment; Stream. 2) Lower Limbs: Stream, Berg Balance scale,10m walk test, timed up\&go test, 6' walk test, Functional Ambulatory Categories.3) Global Functioning: 
Barthel; Functional Independence Measure; Motricity Index; Goal Attainment Scale; QoL scales; International Classification of Functionality and Health; Disability A assessment Scale 4) Instrumented Analysis: gait and movement laboratories, dynamic poly-EMG telemetric systems, baropodography systems, accelerometer based activity measurement systems, robotic evaluation and training devices for upper limb, lower limb, balance/postural control. It's by using the adequate combination of all these evaluation tools/methods for each patient, in each visit/evaluation, that we can get the best possible information, so as to derive a customized multidisciplinary and multimodal treatment plan, which may include botulinum neurotoxin $\mathrm{A}$.

\section{Goal setting and treatment planning}

Before initiating treatment/management of a spastic patient we need to establish treatment goals. This is a complex, but rather compensatory process, that is easy to implement in everyday clinical practice, either for inpatients or outpatients of a specialized spasticity clinic. A few steps are fundamental: firstly, from the anamnesis we find out what the main problems of the patient are that are related to spasticity. Secondly, we select from these problems, those that may be managed by the treatment including botulinum neurotoxin, and we inform the patient what we can do, what he/she should commit to do as well, and what the expected outcomes are. Then, if the patient is interested, these become treatment goals. Goals are therefore identified on an individual basis, negotiated by the rehabilitation team with the patient and/or care takers, and written down according to SMART rules: Specific, Measurable, Achievable, Realistic and Timed. It is recommended to set one primary goal and a few secondary goals, which ideally shouldn't be more than 2-3, for the sake of having both professionals and patient/care taker focused and motivated. Once the time set for follow-up and evaluation of treatment outcomes is reached, health professional team and patient should recur to the set SMART goals and determine if they were achieved and to which extent. This is better accomplished with the use of the GAS (Goal Attainment Scale). GAS is mostly a method for scoring the degree of success in reaching goals during or after some kind of intervention. Patients have personalized measurements for their specific expected outcomes, but the degree of achievement is scored in a standardized way, in order to allow statistical analysis.

\section{Management of spasticity}

Spasticity must be approached and managed by a multidisciplinary specialized team and using a customized multimodal treatment plan, designed to pursuit the goals defined in consensus with the patient and/or care takers. Ideally, in most cases, it should include inputs from the following items.

Physiotherapy: proprioceptive neuromuscular facilitation, balance training, gait training, muscle strengthening, muscle stretching, hydrotherapy, functional electrical stimulation (FES).

Occupational therapy: perceptive-cognitive training, training in domestic daily activities, training for leisure activities, technical aids, games, computer, orthotic fitting and training, wrist and hand mostly, fitting/training on wheelchair positioning and driving.

Activities of daily living (occupational therapy/rehabilitation nurses): hygiene, selfcare/grooming, dressing, bed mobility, transfers (t. board, t.disk, t. elevator), support bars.

Orthotist: Provision of equipment, such as: removable splints to support joints and limbs in positions as physiologic as possible.

Serial casting (doctor + nurse or physiotherapist/occupational therapist): the joint is placed in a plaster cast to prevent contractures and excessive muscle stiffness, sometimes used immediately after botulinum neurotoxin injections.

All these professionals, as well as psychologists and social workers play a crucial role in providing education/advice/advocacy/empowerment to patients and care takers affected by a congenital or acquired condition affecting the central nervous system, of which resulted impairment with spasticity.

\section{Treatment strategies}

Patients should be under the care of a multidisciplinary team, in a specialized neurorehabilitation center, and the team must be experienced in evaluation and treatment of spasticity. Multiple modalities for spasticity management should ideally be available and be used, including: physical agents, physiotherapy, occupational therapy, casts, orthosis, apart from the pharmacological and eventually surgical treatments.

Pharmacological treatments may include systemic medication, which may have some degree of efficacy in these patients. Nevertheless, the side effects, which are deleterious to the functioning of the affected central nervous system, are largely limited to the maximal doses that can be used. In fact, high doses may affect the patients' ability to partici- pate in the physical /cognitive rehabilitation program. Botulinum neurotoxin A is therefore best suited for the treatment of spasticity in patients suffering from spasticity due to cerebral lesions/conditions.

\section{Treating spasticity with botu- linum neurotoxin A: final con- siderations}

The efficacy in reducing muscle tone and the safety profile of BoNT-A, has been largely demonstrated and are nowadays considered level A evidence. There are currently different formulations of BoNT-A in different markets around the world. The units of BoNT-A preparations are not the same and they are not interchangeable. Hence, doses are specific to each preparation, are not interchangeable with each other or with other preparations of botulinum toxin and should not be the basis of efficacy comparisons. Each brand has provided tables with dose recommendations for the approved indications and for the different muscles.

The adverse event profiles have also been widely published, some of the most frequent being: muscle weakness, headache, flu-like symptoms, blurred vision, dry mouth, reduced sweating, constipation/diarrhea, vomiting, urinary incontinence, dysphagia, and gait disturbances, accidental injuries due to falls, pain/soreness/swelling/bruising or bleeding at the puncture site. Most importantly, it's been clearly shown that they are rare, transient and mostly self-limited.

When treating a patient, by definition unique, one major challenge to the physician is to be able to interprete/translate clinical patterns into a list of muscles contributing to the observed pattern and then prioritize the ones to be injected, according to the predefined treatment goals for that injection cycle. There are muscle tables with recommendations for the most frequent patterns, which are easily available in different trustworthy sources (for example, www.mdvu.org). Anesthetic blocks with lidocaine or bupivacaine can be useful tools, as can dynamic poly-emg.

Once the right muscles to inject and the right dose per muscle have been decided, the next step is the injection technique. An accurate technique should guarantee that the toxin is injected in the right muscle and in the vicinity of the greater concentration of motor endplates in each muscle. Several techniques are used around the globe, from simple anatomical landmarks and EMG maps, to what is nowadays considered the best practice in most guidelines, which is the use of one or more targeting techniques. The most popular to date are 
still, EMG and electrical stimulation, but ultrasound and ultra-sound associated to any of the previously mentioned is becoming more and more popular amongst the most experienced and research prone injectors. These physicians/scientists claim, and literature is beginning to show, that efficacy can be increased with lower doses, therefore less cost and less risks of adverse events.

When we think about getting the best results from the treatment, muscle activity must also be considered. It has a direct influence on the response to BoNT-A, as we see a greater degree of nerve block at the more activated muscles. The higher the level of muscle activity, the higher the increase in BoNT-A cellular absorption, thus affecting the amount of neuromuscular blockade. Hence, it's recommended that the injected muscles be mobilized/stretched immediately before and 20-30 minutes after the injection.

This is a medication used in spasticity treatment for more than 20 years, and yet, relevant questions are still lacking consensual answers from available scientific literature and key opinion leaders around the world. Namely, how much does this treatment modality improve functionality in a way that can influence activities performance, or reduce care taker need, or improve social participation, or quality of life ? When should treatment be started and stopped? Who are the golden responders? And, of course is its cost effective in the long term? Pools of studies have started to be developed in the last 10 years, and some are already published, nevertheless international guidelines are still unclear in some of these issues.

\section{References}

1. Garcia-Ruiz PJ. Applications of botulinum toxin in Neurology. Med Clin (Barc) 2013;141:33-6.

2. Rosales RL, Arimura K, Takenaga S, Osame M. Extrafusal and intrafusal muscle effects in experimental botulinum toxin-A injection. Muscle Nerve 1996;19:488-96.

3. Singer C. Indications and management of botulinum toxin. Rev Neurol 1999;29:157-62.

4. Mayer NH. Clinicophysiologic concepts of spasticity and motor dysfunction in adults with an upper motoneuron lesion. In: Mayer NH, Simpson DM, eds. Spasticity: etiology, evaluation, management, and the role of botulinum toxin. New York: Wemove; 2002. pp 1-10.
5. Wissel J, Ward AB, Erztgaard P, et al. Consensus table on the use of botulinum toxin type A in adult spasticity. J Rehabil Med 2009;41:13-25.

6. Mayer NH, Simpson DM. Patient encounter forms and rating scales. Appendix. Spasticity examination rating scales. In: Mayer NH, Simpson DM, eds. Spasticity: etiology, evaluation, management, and the role of botulinum toxin. New York: Wemove; 2002.

7. Mahoney FI, Barthel DW. Functional evaluation: Barthel index. Md State Med J 1965;14:61-5.

8. Keith RA, Granger CV, Hamilton BB, Sherwin FS. The functional independence measure: a new tool for rehabilitation. Adv Clin Rehabil 1987;1:6-18.

9. Graham HK, Selber P. Musculoskelectal aspects of cerebral palsy. J Bone Joint Surg Br 2003;85:157-66.

10. Davis TL, Brodsky MA, Carter VA, et al. Consensus statement on the use of botulinum neurotoxin to treat spasticity in adults. Available from: http://www.pharmscope.com/system/files/PTJ3111666.pdf.

11. Jankovic J, Brin MF. Botulinum toxin: historical perspective and potential indications. Muscle Nerve Suppl 1997;6:S129-45.

12. Truong D, Dressler D, Hallett M. Manual of botulinum toxin therapy. Cambridge: Cambridge University Press; 2009. p 267.

13. Lima CLA, Fonseca LF. Paralisia cerebral. Rio de Janeiro: Guanabara Koogan; 2004. p 55.

14. Brin MF. Treatment of spasticity using local injections of botulinum toxin. Skills Workshop Series Seattle: American Academy of Neurology, 1995.

15. Otom AH, Al-Khawaja IM, Al-Quliti KW. Botulinum toxin type-A in the management of spastic equinovarus deformity after stroke. Comparison of 2 injectiontechniques. Neurosciences (Riyadh) 2014;19:199-202.

16. Castro-Costa CM, Araújo AQC, Barreto MM, et al. Guia de Manejo Clinico do paciente com HTLV: aspectos neurológicos. Arq Neuro-Psiquiatr 2005;63:5548-51.

17. Stokholm MG, Bisgard C, Vilholm OJ. Safety and administration of treatment with botulinum neurotoxin for sialorrhoea in ALS patients: review of the literature and a proposal for tailored treatment. Amyotroph Lateral Scler Frontotemporal Degener 2013;14:516-20.

18. Restivo DA, Casabona A, Nicotra A, et al. ALS dysphagia pathophysiology: differential botulinum toxin response. Neurology 2013;80:616-20.
19. Mezaki T, Kaji R, Kohara N, et al. Development of general weakness in a patient with amyotrophic lateral sclerosis after focal botulinum toxin injection. Neurology 1996;46:845-6.

20. Koussoulakos S. Botulinum neurotoxina: the ugly duckling. Eur Neurol 2009;61:33142.

21. Koman LA, Smith BP, Williams R, et al. Upper extremity spasticity in children with cerebral palsy: a randomized, double-blind, placebo-controlled study of the short-term out comes of treatment with botulinum A toxin. J Hand Surg Am 2013;38:435-46.

22. Koog YH, Min BI. Effects of botulinum toxin A on calf muscles in children with cerebral palsy: a systematic review. Clin Rehabil 2010;24:685-700.

23. Chen S. Clinical uses of botulinum neurotoxins: current indications, limitations and future developments. Toxins 2012;4:913-39.

24. Ramirez-Castaneda J, Jankovic J. Long term efficacy and safety of botulinum toxin injections in dystonia. Toxins 2013;5:249-66.

25. Ramirez-Castaneda J, Jankovic J. Longterm efficacy, safety, and side effect profile of botulinum toxin in dystonia: a 20-year follow-up. Toxicon 2014;90:344-8.

26. Colhado OCG, Boeing M, Ortega LB. Botulinum toxin in pain treatment. Rev Bras Anestesiol 2009;59:366-81.

27. Jackson JL, Kuriyama A, Hayashino Y. Botulinum toxin A for prophylactic treatment of migraine and tension headaches in adults. A meta-analysis. JAMA 2012;307:1736-45.

28. Lakraj AA, Moghimi N, Jabbari B. Sialorrhea: anatomy, pathophysiology and treatment with emphasis ont he role of botulinum toxins. Toxin 2013;5:1010-31.

29. Vashishta R, Nguyen SA, White DR, Gillespie MB. Botulinum toxin for the treatment of sialorrhea: a meta-analysis. Otolaryngol Head Neck Surg 2013;148:1916.

30. Nigam PK, Nigam A. Botulinum toxin. Indian J Dermatol 2010;55:8-14.

31. Yelnik AP, Simon 0, Parratte B, Gracies JM. How to clinically assess and treat muscle overactivity in spastic paresis. J Rehabil Med 2010;42:801-7.

32. Bakheit AM, Fheodoroff K, Molteni F. Spasticity or reversible muscle hypertonia? J Rehabil Med 2011;43:556-7. 